\title{
Designing a Mobile Video Game to Help Young Deaf Children Learn Auslan
}

\author{
Jessica Korte \\ School of ICT \\ Griffith University \\ 170 Kessels Rd, Nathan, Q 4113 \\ Australia \\ j.korte@griffith.edu.au
}

\author{
Leigh Ellen Potter \\ School of ICT \\ Griffith University \\ 170 Kessels Rd, Nathan, Q 4113 \\ Australia \\ I.potter@griffith.edu.au
}

\author{
Sue Nielsen \\ School of ICT \\ Griffith University \\ 170 Kessels Rd, Nathan, Q 4113 \\ Australia \\ s.nielsen@griffith.edu.au
}

\begin{abstract}
Hearing-impaired children of hearing parents face challenges in learning sign languages, due in part to a lack of language immersion. These difficulties are compounded by the lack of learning resources available to these children. Technological resources, in particular, have the potential to aid Deaf children learning sign languages. The increasing ubiquity of mobile devices such as smart phones and tablets presents an opportunity for the development of such resources which could be made available to a wide audience, while also enabling developers to utilise mobile-specific features such as context awareness. The Seek and Sign project aims to create mobile-delivered technological resources to aid Deaf children learning Australian Sign Language (Auslan), such as the Sign My World application. This paper will discuss early work in the development of this application, with a focus on the potential uses of video game principles, context awareness and system personalisation in promoting learning, and consideration of how they may be implemented in Sign My World. We will present an interface prototype, as well as a briefly outlining a series of requirements elicitation sessions undertaken to discover a direction for future developments of the application.
\end{abstract}

Participative design, Child Computer Interaction, Deaf Children, Context Awareness, Personalisation, eLearning Game

\section{INTRODUCTION}

A significant majority of hearing-impaired children are born to hearing parents, and these children frequently face a disadvantage when it comes to learning sign language. Unlike hearing children or the children of Deaf parents who are already able to sign, these children are not immersed in language during their early life. Delayed exposure to first language has been shown to cause lifelong difficulties in comprehension and fluency (Kyle, 2009; Mayberry \& Eichen, 1991).

Achieving the solution - early immersion in sign language - can be difficult for hearing parents who are unfamiliar with sign languages. Current techniques include extensive, expensive teaching sessions for the family with a fluent signer, with parents able to offer their hearing-impaired children minimal assistance as they learn the language alongside their child.

Technological resources have the potential to be invaluable to these children and their families in learning sign languages. There are numerous characteristics of multimedia applications and video games which lend themselves to learning, particularly when dealing with sign languages. Displaying signs through videos and animations provides details that books and posters simply cannot match with static images.

The goal of the Seek and Sign research project is to create technological resources to aid these children in the acquisition of Australian Sign Language (Auslan), such as the development of the Sign My World proof of concept described in this paper. As part of this project, the capabilities of mobile technologies, eLearning and video game principles are being explored for their suitability for inclusion within an information technology resource to enhance learning.

This paper briefly outlines recent attempts at creating technological sign language learning resources for young children. Three important concepts for this resource are explored: context awareness, personalisation, and video game principles. Following this, an initial proof of concept is described, along with the aspects of context awareness, personalisation and video game principles which are being examined for feasibility. 
Next there follows a discussion of a series of prototype review sessions, which were undertaken to elicit user requirements for development of the prototype. Finally, the future work and the implications of this research are explored.

\subsection{The difference between 'Deaf' and 'deaf'}

There is a convention of capitalisation when discussion deaf and hearing-impaired individuals. A person is 'deaf' if they have a severe hearing impairment. In this paper, 'deaf' may be used synonymously with 'hearing impaired'. A 'Deaf' person is a member of Australia's Deaf community who values and uses Auslan. The Deaf community, like various ethnic communities, has its own language (Auslan) and culture, which still belonging to the wider Australian community.

\section{RELATED WORK}

Very little work has been undertaken to explore the use of technology to support learning resources for teaching Auslan. Ellis has undertaken work to support young Deaf children (Ellis, 2007) and has recently completed a software development to teach Auslan to hearing people (Ellis, Ray \& Howard, 2011), however little else exists in Australia. Preliminary interviews conducted for this project suggest that most of the available Auslan technology-based resources are designed to teach hearing and/or literate learners, such as the Royal Institute for Deaf and Blind Children Auslan Tutor. Those resources which are designed for preliterate Deaf children have been created in schools on an ad-hoc basis, and require the guidance of an adult or a fluent signer.

A survey of resources for very young children learning sign languages found that the development of resources for American Sign Language (ASL) is far more prolific than those for Auslan; for example, researchers at the Georgia Institute of Technology have developed CopyCat (Henderson et al., 2005), the iSign Bear (Huang et al., 2008) and PlayWare (Yarosh et al., 2008). However, translating such resources into Auslan would be expensive and time consuming. The best case scenario requires replacement of all ASL videos with their Auslan counterparts. Furthermore, software which responds to signed user input, such as CopyCat, would require reprogramming of the sign recognition functionality. Auslan has some similiarities to British Sign Language (BSL), but is significantly different from ASL and many other sign languages. When these difficulties are considered, it becomes clear that a need exists for the creation of Auslan specific resources.

\section{KEY CONCEPTS FOR THE SIGN MY WORLD APPLICATION}

The Seek and Sign research project aims to create a game-based eLearning resource called Sign My World using a user centred design approach, for delivery on a mobile device, which will assist preliterate Deaf children in learning Auslan by allowing them to become familiar with the appearance of common noun and verb signs.

Key concepts of the investigation include:

- How the context awareness aspect of mobile computing can be incorporated into the product to enhance learning.

- How personalisation can enhance learning.

- How a video game-style interface can be used to engage learners.

As mobile computing becomes more ubiquitous, context awareness is an increasingly important area of consideration. It allows applications to increase their relevance to users by modifying functionality, appearance or content based on the user's context. Context can be described as the aspects of the situation the user and application are operating in, which have an impact on the performance of the user or application, specifically regarding questions of who, where, when and what (Adomavicius \& Tuzhilin, 2011; Baldauf, Dustdar \& Rosenberg, 2007; Bolchini et al., 2007; Chen \& Kotz, 2000; Dey \& Abowd, 2000; Hexel et al., 2004). Context awareness functionality has the potential to expand the scope and capabilities for the Sign My World application.

Individuals learn more easily when information, and the way in which it is presented, is suited to their prior learning, preferences, and habits (Hexel et al., 2004; Kay, 2008). Personalisation in this context refers to the ability of a software application to customise the information and features provided to an individual. The features offered through personalisation of content may enhance the learning experience for children using the Sign My World application.

Many researchers have recognised the potential for video games to contribute to learning. An effective video game offers players an immersive, interactive experience which entertains and rewards progress, while allowing the player to set their own pace in working toward clearly-defined goals. (Barab et al., 2005; Bourgonjon et al., 2011; Gee, 2007) In an educational game, this translates to learners who are self-motivated to continue playing until they have completed the game, thus acquiring and demonstrating important skills and knowledge. Exposure to video games has been shown to enhance physical abilities, including visual acuity, 
motor coordination and spatial awareness - all important skills when communicating in a gestural language - and mental abilities, such as numerical concepts, reading comprehension, deep learning and creative thinking (de Aguilera \& Mendiz, 2003). The inclusion of video game aspects in the Sign My World application may be useful in harnessing these benefits.

\section{THE PROOF OF CONCEPT}

A proof of concept was designed to test the feasibility of incorporating context awareness, personalisation and video game principles in a learning tool. A working proof of concept was created, as opposed to a paper-based prototype, as this provided the greatest opportunity to examine the actual effects of the concepts. Aspects of all three concepts were incorporated into a single proof of concept in order to test for any conflicts; however, this study was not intended to examine the interactions between instances of the concepts, and further work would be required to examine this.

Although the final product is intended for mobile devices such as phones and tablets, the proof of concept was developed on a laptop device. This choice was made in order to allow easier manipulation of the design elements during development, and to facilitate a simpler interaction between young Deaf children and the development team during requirements elicitation sessions. Observations and user comments during these sessions were used to guide development of the prototype.

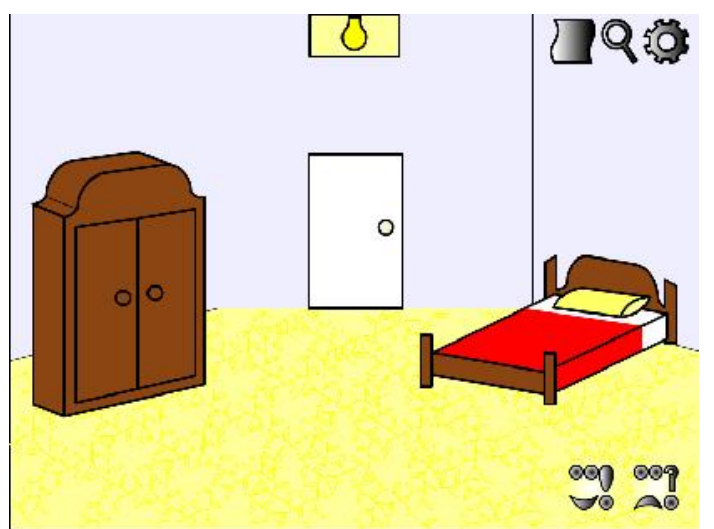

Figure 1: The proof of concept interface began as a bedroom area which users can explore to reveal Auslan signs. The parental control panel (top right corner) consists of dummy buttons (progress report, search, settings) for future development. The user's control panel (bottom right corner) has a tutorial button, and a dummy testing button.

The proof of concept interface has a 2D, cartoonlike style and bright colours (shown in Figure 1) as this was determined to be appealing to young children (Light, Drager \& Nemser, 2004) . It began as a single sample area - a bedroom - containing a number of interactive objects. When an interactive object is clicked, an image and word are displayed, as on a flash card; followed by the video of the Auslan sign for that object. This is intended to allow the child to make associations between the object and the sign.

The position of a sign can be important to its meaning in Auslan. Due to this, the video displays at least the signer's upper body and head, as shown in Figure 2. In some cases, it will be necessary to display more of the signer's body, or to display it from a different angle, to show the correct position, and those videos will be framed appropriately. However, videos which allow the signer's face to be clearly visible are preferred. This is important, as the signer will speak the sign's English word as they sign it. This provides another method for a child with some hearing or lip-reading ability to form an association between the sign and the English word. It is largely for this reason that videos were chosen over animated characters. It could be both difficult to program the fine detail of realistic finger movements, facial expression and mouth shape on an animated character. An attempt to do so could even invoke the uncanny valley effect (Mori, 1970). Initially, a number of Auslan videos included fingerspelling, the spelling of an English word using Auslan letter signs, which further increased the difficulty of creating realistic animations, both in the display of signs and the transitions between them.

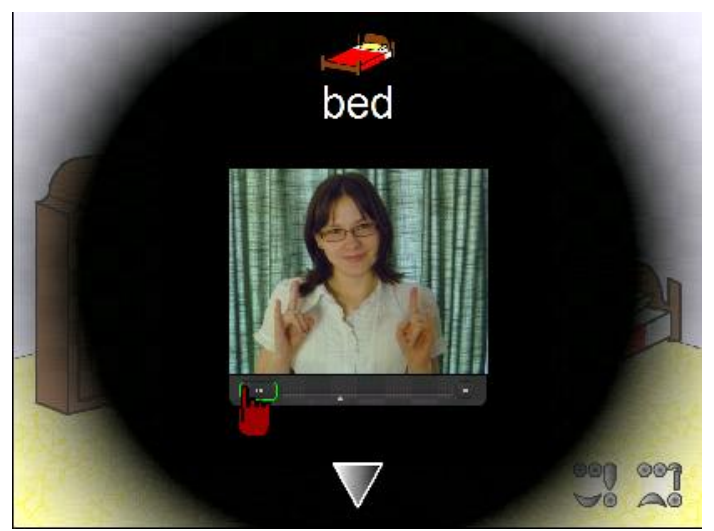

Figure 2: The flash card image and word remain visible as the sign video plays, though the image's size is reduced.

Some items in the proof of concept had a second, more difficult 'level' of signs available to them - in this case, verbs (for example, "open door") - which could be unlocked to keep the child's interest after they have learnt the 'easier' words.

The development of the proof of concept on a laptop, rather than a phone or tablet-style mobile 
device impaired the implementation of the context aware features. A simple example was implemented to test feasibility of incorporating context awareness into the Sign My World application. The proof of concept uses time context to determine the likelihood of adult supervision. An adult presence, in this case, was used to hide or reveal the parental controls (top right corner of figure 1); however, this information could also be used to unlock more difficult words/signs and concepts while an adult is available to help. Adult availability was determined by time of day, day of the week, and the assumption that an adult would be present during non-work hours (i.e. outside of 9 a.m. to 5 p.m. on weekdays). This was chosen as a feasibility test for time context awareness. Had a settings control panel been implemented, expected adult availability would have been included as a customisable setting. Further investigation of context awareness will be undertaken in future developments.

Automatic interface personalisation has been implemented to allow the proof of concept to adapt to the user's patterns of behaviour. When viewing the 'flash card' for a sign, there are behaviours the system can 'learn'. The system tracks the number of times the flash card image and the video are viewed. If the user views images, on average, more than twice, the image will be enlarged after the first play-through of the video. If the user plays videos, on average, two or more times, the video will be automatically repeated. This could be particularly useful for children attempting to mimic the sign shown, as they will not need to reach for the mouse mid-sign. Further investigation will examine the relevance of this behaviour to the users.

Some simple implementations of user-controlled personalisation have also been included. A parental control panel is available, which allows users or their parents to select the availability of the extra level of signs, and which could be used to personalise other aspects of the prototype. A 'character creation' screen was created, which could be used to personalise a user avatar, or other human characters in the prototype.

As the aim of this implementation is proof of concept only, these are rather simple uses of personalisation and user login or identification facilities have not been included. These would be necessary for user-specific personalisation.

The proof of concept provides self-pacing, exploration and interactivity due to the open nature of the application. A pair of sign-to-image matching games were incorporated into the prototype. These Further development will focus on enhancing the game-like nature of the main application, to increase user engagement and learning potential.

\section{REQUIREMENTS ELICITATION SESSIONS AND FUTURE DEVELOPMENTS}

A series of requirements elicitation sessions, in the form of prototype testing sessions, were conducted with three seven-year-old boys. One participant was profoundly deaf, had a cochlear implant, and experienced delayed language development. The other two were able to hear with the use of hearing aids, and were learning both Auslan and vocalised English. The results of these sessions were used to outline possible future development of the Sign My World application

During sessions, participants were provided with a laptop displaying the proof of concept and asked to 'play' with the game during a 15 to 30 minute session with the developer and an Auslan interpreter. Observations were made based on his activities and reactions, and participant comments were recorded in writing. Based on observations made during sessions and the participants' comments, a number of developments were made to the prototype between sessions, including the creation of new areas and word sets.

Participants seemed to enjoy the bright colours and cartoon-like aspect of the interface, and one stated that he "loved" the animated buttons. It became a point of concern for two participants, during a later session, when some new items were not animated. One participant was almost immediately able to discern that the animated items could be clicked on; the other two were more hesitant about clicking on items.

During the first testing session, a participant tried to return to the main area from a flash card by clicking on the faded black/transparent area behind the video (shown in Figure 2). This had no effect, which frustrated him, until he noticed and clicked the 'back' button. Due to this, the 'back' button was removed from the prototype, and the flash card background became a clickable area which returned users to the main area.

The inclusion of fingerspelling in the Auslan videos proved to be confusing to participants. In future developments, this should be removed. Perhaps as an aspect of personalisation, it could be restored with a user-selected option.

Participants indicated that they enjoyed interacting with the prototype, and seemed to be learning the incorporated signs after multiple sessions.

There were a number of features of the prototype that participants did not examine during requirements elicitation sessions. The tutorial button remained untouched. Either its meaning was unclear to participants, or they did not feel they 
needed help in navigating the interface. Item images were never re-viewed, and the unlocking of verbs for some items went unremarked. This may have been due to the placement of the item- and verb images on the flash card, above the English word.

Future developments of the application must be implemented for delivery and testing on mobile devices, in order to fully explore the opportunities provided by context awareness. There are numerous possibilities for improvement with the implementation of further context awareness, personalisation and video game features.

Context awareness could be used in promoting context-relevant lessons. Location could be used to determine the most relevant set of objects; "register", "money" and "shopkeeper" could display automatically if the user is at the shops. Time of day could be used to expand or change the set of verbs and related words; for example, "Make bed" in the morning versus "sleep" at night. Time of the year could alter objects appearing in sets, such as emphasizing summer or winter clothing during those seasons.

It would also be possible to determine the availability of learning resources or adult assistance from other aspects of context, including:

- Location - if resources are known to exist in a certain location, such as "at home" or "at school".

- Movement - if the user seems to be travelling, they are not likely to have resources available to them beyond those already on the mobile device in use.

- Network connection - if certain networks can be identified as "home", "school", etc, it would suggest that resources or adult help would be available.

- Detection of other nearby computing devices - such as a parent's mobile device or computer, which might suggest adult availability.

Personalisation could be used to enhance the specific relevance of the application to the individual using the application. At present, the determining factor in deciding when to unlock the next level of words is simply the number of interactions between user and application. In future implementations, this could be based on user success rate in a game or test. User identification, such as a login, would be required for individual personalisation in a multi-user situation, allowing each user to progress at their own pace. This is an option to consider for future development.

Personalisation can also be used in reporting the learner's progress to their guardians. The application would be able to generate reports, containing different subsets of information about the learner's progress, for parents and teachers.

Video game principles will be implemented in such a way as to emphasise creativity and problem solving, two aspects game play prevalent in activities participants identified as 'fun'. Context awareness and personalisation concepts can also be used to adapt games/tests. The scope and difficulty of game/s could be adjusted (personalised) based on the learner's skill and experience, or the availability of resources (context). The content or focus of the game/s could also be determined by context.

\section{CONCLUSION}

This project has the potential to help a disadvantaged and often-overlooked group. The purpose of this resource is to expose preliterate Deaf children to Auslan in a fun and engaging way, which will provide an opportunity for greater immersion in their first language. This early immersion has the potential to enhance comprehension and literacy among Deaf children.

This paper reflects only the first steps into an under-explored but important area. The proof of concept presented here serves as a cursory examination of the feasibility of incorporating context awareness, personalisation and video game principles in learning resources to engage learners and enhance learning.

\section{REFERENCES}

Adomavicius, G. \& Tuzhilin, A. (2011) ContextAware Recommender Systems. In: Francesco Ricci, Lior Rokach, Bracha Shapira, \& Paul B. Kantor (eds.). Recommender Systems Handbook. Springer US, Boston, MA. pp. 217-253.

de Aguilera, M. \& Mendiz, A. (2003) Video Games and Education (Education in the Face of a "Parallel School"). Computers in Entertainment. 1 (1), 1-14.

Baldauf, M., Dustdar, S. \& Rosenberg, F. (2007) A survey on context-aware systems. International Journal of Ad Hoc and Ubiquitous Computing. 2 (4), 263-277.

Barab, S., Thomas, M., Dodge, T., Carteaux, R. \& Tuzun, H. (2005) Making learning fun: Quest Atlantis, a game without guns. Educational Technology Research and Development. 53 (1), 86-107. 
Bolchini, C., Curino, C.A., Quintarelli, E., Schreiber, F.A. \& Tanca, L. (2007) A data-oriented survey of context models. AGM SIGMOD Record. 36 (4), 19-26.

Bourgonjon, J., Valcke, M., Soetaert, R., de Wever, B. \& Schellens, T. (2011) Parental acceptance of digital game-based learning. Computers \& Education. 57 (1), 1434-1444.

Chen, G. \& Kotz, D. (2000) A survey of contextaware mobile computing research. p.1-16.

Dey, A.K. \& Abowd, G.D. (2000) Towards a better understanding of context and contextawareness. In: Proceedings of CHI 2000. April 2000 The Hague, The Netherlands. pp. $1-12$.

Ellis, K. (2007) Multimedia to assist kindergarten children to learn Australian sign language. Journal of Australian Research in Early Childhood Education. 14 (2), 12.

Ellis, K., Ray, N. \& Howard, C. (2011) Learning a physical skill via a computer: a case study exploring Australian Sign Language. In: Proceedings of OZCHI 2011. December 2011 Canberra, Australia. pp. 98-103.

Gee, J.P. (2007) Good Video Games + Good Learning. Peter Lang Publishing, New York, New York, USA.

Henderson, V., Lee, S., Brashear, H., Hamilton, H., Starner, T. \& Hamilton, S. (2005) Development of an American sign language game for deaf children. In: Proceedings of IDC 2005. June 2005 pp. 70-79.
Hexel, R., Johnson, C., Kummerfeld, B. \& Quigley, A. (2004) "PowerPoint to the People": Suiting the Word to the Audience. In: Proceedings of the fifth conference on Australasian user interface. January 2004 Australian Computer Society, Inc., Dunedin, New Zealand. pp. 4956.

Huang, K., Smith, J., Spreen, K. \& Jones, M.F. (2008) Breaking the sound barrier: designing an interactive tool for language acquisition in preschool deaf children. In: Proceedings of IDC 2008. September 2008 pp. 210-216.

Kay, J. (2008) Lifelong Learner Modeling for Lifelong Personalized Pervasive Learning. IEEE Transactions on Learning Technologies. 1 (4), 215-228.

Kyle, J. (2009) Deaf children learning to sign. Educação Temática Digital. 2 (3), 27-37.

Light, J.C., Drager, K.D. \& Nemser, J.G. (2004) Enhancing the Appeal of AAC Technologies for Young Children: Lessons from the Toy Manufacturers. Augmentative and Alternative Communication. 20 (3), 137-149.

Mayberry, R.I. \& Eichen, E.B. (1991) The longlasting advantage of learning sign language in childhood: Another look at the critical period for language acquisition. Journal of Memory and Language. 30 (4), 486-512.

Mori, M. (1970) The Uncanny Valley. Energy. 7 (4) 33-35.

Yarosh, S., Huang, K., Mosher, I. \& Topping, M. (2008) Playware: augmenting natural play to teach sign language. In: Proceedings of $\mathrm{CHI}$ 2008. April 2008 pp. 3249-3254. 